\title{
ADVANCED LIPOPROTEIN MEASURES AND RECURRENT PRETERM BIRTH
}

\author{
John M. Thorp Jr., M.D., Madeline Murguia Rice, Ph.D., Margaret Harper, M.D., M.Sc., Mark \\ Klebanoff, MD, MPH, Yoram Sorokin, M.D., Michael W. Varner, M.D., Ronald J. Wapner, \\ M.D., Steve N. Caritis, M.D., Jay D. lams, M.D., Alan M. Peaceman, M.D., Brian M. Mercer, \\ M.D., Anthony Sciscione, D.O., Dwight J. Rouse, M.D., Susan M. Ramin, M.D., Garland B. \\ Anderson, M.D., and for the Eunice Kennedy Shriver National Institute of Child Health and \\ Human Development Maternal-Fetal Medicine (MFMU) Units Network ${ }^{*}$ \\ Departments of Obstetrics and Gynecology of University of North Carolina, Chapel Hill, NC \\ (J.M.T.); Wake Forest University Health Sciences, Winston-Salem, N.C. (M.H.); Wayne State \\ University, Detroit, MI (Y.S.); University of Utah Health Sciences Center, Salt Lake City, UT \\ (M.W.V.); Columbia University, New York, NY (R.J.W.); University of Pittsburgh, Pittsburgh, PA \\ (S.N.C.); The Ohio State University, Columbus, OH (J.D.I.); Northwestern University, Chicago, IL \\ (A.M.P.); Case Western Reserve University-MetroHealth Medical Center, Cleveland, $\mathrm{OH}$ \\ (B.M.M.); Drexel University College of Medicine, Philadelphia, PA (A.S.); University of Alabama at \\ Birmingham, Birmingham, AL (D.J.R.); University of Texas Health Science Center at Houston, \\ Houston, TX (S.M.R.), University of Texas Medical Center, Galveston, TX (G.B.A); and The \\ George Washington University Biostatistics Center, Washington, DC (M.M.R.); and the Eunice \\ Kennedy Shriver National Institute of Child Health and Human Development, Bethesda, MD \\ (M.K.)
}

\section{Abstract}

OBJECTIVE-Lipoproteins are associated with atherogenic and inflammatory processes, and these processes may be related to adverse pregnancy outcomes. We therefore examined whether variations in lipoprotein particle size and concentration are associated with preterm birth (PTB) < 35 weeks' gestation.

\begin{abstract}
METHODS-This is a case-control ancillary study to a randomized trial of omega-3 fatty acid supplementation to prevent recurrent PTB. We measured standard lipids and used nuclear magnetic resonance (NMR) spectroscopy to characterize 17 lipoprotein particles from plasma collected at the baseline randomization visit (16-21 weeks gestation) in 128 cases (PTB < 35 weeks' gestation) and 132 term controls. Logistic regression models controlled for study center, race/ethnicity, number of prior PTB, smoking and treatment group, as well as total LDL, HDL and triglyceride concentrations when examining $\mathrm{LDL}_{\mathrm{NMR}}$ lipoproteins, $\mathrm{HDL}_{\mathrm{NMR}}$ lipoproteins and VLDL $_{\text {NMR }}$ lipoproteins, respectively.
\end{abstract}

\footnotetext{
() 2013 Mosby, Inc. All rights reserved.

* Other members of the Eunice Kennedy Shriver National Institute of Child Health and Human Development Maternal-Fetal Medicine Units Network are listed in the Appendix

Publisher's Disclaimer: This is a PDF file of an unedited manuscript that has been accepted for publication. As a service to our customers we are providing this early version of the manuscript. The manuscript will undergo copyediting, typesetting, and review of the resulting proof before it is published in its final citable form. Please note that during the production process errors may be discovered which could affect the content, and all legal disclaimers that apply to the journal pertain.

The following MFMU Network members participated in protocol development and coordination between clinical research centers (Karen Dorman, R.N., M.S.), protocol/data management and statistical analysis (Paula McGee, M.S. and Elizabeth Thom, Ph.D.), and protocol development and oversight (Catherine Y. Spong, M.D.).
} 
RESULTS-Only one of the 17 NMR lipoproteins was associated with recurrent PTB. We observed an increased odds of recurrent PTB of $1.04(95 \% \mathrm{CI}=1.01-1.08 ; \mathrm{p}=0.02)$ per nanometer increase in $\mathrm{VLDL}_{\mathrm{NMR}}$ particle size and an odds ratio of $3.00(\mathrm{CI}=1.40-6.43 ; \mathrm{p}=0.005)$ for the $3^{\text {rd }}$ tertile of VLDL $\mathrm{VMR}_{\mathrm{N}}$ particle size compared with the $1^{\text {st }}$ tertile.

CONCLUSION-In women with prior PTB, variations in mid-pregnancy lipoproteins were not associated with recurrent PTB overall, however the trend observed with VLDL NMR $_{\text {particle size is }}$ suggestive that PTB may be amenable to lifestyle, nutritional or pharmacologic interventions.

\section{INTRODUCTION}

The adaptation of a mother to pregnancy culminates in the mobilization of fatty acids from maternal fat stores in response to increases in insulin resistance that peaks and plateaus at mid-gestation (1). Pregnancy-associated insulin resistance is induced by pregnancy hormones and is associated with higher fasting plasma triglycerides (TG) and lower high density lipoprotein concentrations (HDL) (2). Perturbations in this response are more pronounced in obese women $(2,3,4)$, are associated with increases in inflammatory markers $(1,2)$, and are associated with adverse outcomes in pregnancy, such as gestational diabetes and preeclampsia $(5,6)$. Moreover, these insulin resistance induced metabolic and inflammatory changes can enzyme expression in maternal adipose tissue and be associated with preterm birth (PTB) (6).

Lipoprotein perturbations are causal factors in an array of atherogenic and inflammatory diseases $(7,8)$. Given that inflammation and vascular compromise are hypothesized as causal paths culminating in PTB, and that pregnancy profoundly alters lipid metabolism, it is reasonable to assume that lipoprotein changes might be associated with an increased likelihood of PTB $(9,10)$. We therefore conducted this investigation to determine whether lipoprotein particle size and number (concentration) are associated with recurrent PTB using an advanced lipoprotein measure, nuclear magnetic resonance spectroscopy (NMR), which allows one to measure lipoprotein particle size and concentration (11).

\section{MATERIALS AND METHODS}

The data for this report are from the Eunice Kennedy Shriver National Institute of Child Health and Human Development Maternal-Fetal Medicine Units (MFMU) Network randomized clinical trial of omega-3 long chain polyunsaturated fatty acid (LCPUFA) supplementation to prevent recurrent PTB. The trial was conducted at 13 Network Centers from January, 2005 to October, 2006 and recruited women who had a history of at least one previous spontaneous singleton PTB (12). A total of 434 women were randomized to receive daily supplementation of $1200 \mathrm{mg}$ eicosapentaenoic acid (EPA, 20:5n-3) and $800 \mathrm{mg}$ of docosahexaenoic acid (DHA, 22:6n-3); while 418 were assigned to matching placebos, beginning at $16^{0}$ to $21^{6}$ weeks' gestation and continuing until $36^{6}$ weeks' gestation or delivery, whichever occurred first. As part of the trial, all enrolled women also received weekly injections of 17 alpha-hydroxyprogesterone caproate. Women currently taking fish oil or omega-3 PUFA supplements were ineligible for the trial; detailed inclusion and exclusion criteria are reported elsewhere (12). Gestational age at delivery was available for all 852 participants. The study (NCT00125902 at www.clinicaltrials.gov) was approved by the IRBs of all participating centers and this secondary analysis was determined to be exempt from IRB review by the Office of Human Subjects Research by the University of North Carolina, Chapel Hill NC IRB office. All enrolled women gave written informed consent for participation in the primary study (12). Eligibility for this secondary analysis was restricted to participants consenting to the use of their blood for future research on prematurity and other pregnancy complications. 
The current analysis is a nested case-control study in which patients that delivered $\geq 37$ weeks' gestation were selected as controls and matched on race/ethnicity and study center in an approximate 1:1 ratio to cases, defined as delivery before 35 weeks' gestation. Gestational age at birth was determined from the sonographically-confirmed gestational age at randomization and the elapsed time from randomization to delivery.

Blood was collected at the baseline randomization visit ( $16^{0}$ to $21^{6}$ weeks' gestation), before dispensing study drug. Subjects were not instructed to fast. Standard lipids were measured by Lipoprofile using a nuclear magnetic resonance autoanalyzer and included total cholesterol, LDL cholesterol, HDL cholesterol and triglycerides. We relied on a commercially available laboratory process (Liposcience ${ }^{\circledR}$, Raleigh, NC) that uses nuclear magnetic resonance (NMR) technology to assess each individuals' lipoprotein particle concentration and size, including very low density lipoprotein (VLDL), low density lipoprotein (LDL), and high density lipoprotein (HDL) classes and subclasses (NMR Lipoprofile test [Registered Trademark]). Nuclear magnetic resonance allows investigators to forego the high expense and labor-intensive approach of ultracentrifugation and has been used in over 1,000 clinical trials and cohort studies $(13,14)$. In this study, NMR spectroscopy was used to characterize particle size (in nanometers) and number (concentration in particle mol/l) of 17 lipoprotein particles from plasma. Particle size categorization was done using parameters previously delineated using this technology.

Prior to conducting the multivariable analysis, to determine whether the association between each lipid biomarker and recurrent PTB $<35$ weeks' gestation was linear, we assessed the lipid biomarkers as continuous variables in a model free manner by applying a local smoother (LOESS). The patients were ranked by lipid value to create 10 groups. For each group, the median lipid value was calculated along with the corresponding log(odds) of recurrent PTB. These points were plotted and fitted with two non-parametric (LOESS) smoothers with 2 separate bandwidths ( 0.5 and 1.0) and the linearity in the $\log$ (odds) was assessed. Where there was evidence of non-linearity, and when assessing a lipid biomarker as a continuous variable in a logistic model, we included both linear and quadratic terms for the lipoprotein.

The association between the lipid biomarkers and recurrent PTB <35 weeks' gestation was assessed using logistic regression, conditional on race/ethnicity (Hispanic, non- Hispanic Black, non- Hispanic White) and study center and adjusting for treatment group. The following clinically relevant variables were assessed for confounding: age, number of prior preterm deliveries (1,2 or more), smoking status and pre-pregnancy body mass index (kg/ $\mathrm{m}^{2}$ ). To assess whether the association between NMR lipoproteins and recurrent PTB was independent of standard lipids, models were also adjusted for LDL, HDL and triglycerides when examining $\mathrm{LDL}_{\mathrm{NMR}}$ lipoproteins, $\mathrm{HDL}_{\mathrm{NMR}}$ lipoproteins and $\mathrm{VLDL}_{\mathrm{NMR}}$ lipoproteins, respectively; the collinearity between these variables was first assessed. The lipid biomarkers were assessed as continuous variables, including a quadratic term when relevant, and also divided into tertiles based on the distribution of the controls as an alternate approach to present associations. We also assessed whether the association between the NMR lipoproteins and recurrent PTB differed between the treatment groups by including NMR lipoprotein $\times$ treatment group interaction terms in the multivariable logistic models. The Hosmer-Lemeshow test was used to check for model fit. All analyses were two-sided and a p-value $<0.01$ was considered statistically significant.

\section{RESULTS}

The characteristics of the study population are shown in Table 1. The women with recurrent PTB were more likely to have smoked during pregnancy and to have had two or more 
previous PTBs. Table 1 also provides the medians and interquartile ranges for the midpregnancy lipid biomarkers. Multivariable models controlled for race/ethnicity, center, treatment group, number of prior preterm deliveries and smoking status; $\mathrm{LDL}_{\mathrm{NMR}}$ lipoproteins were also adjusted for LDL cholesterol; $\mathrm{HDL}_{\mathrm{NMR}}$ lipoproteins were also

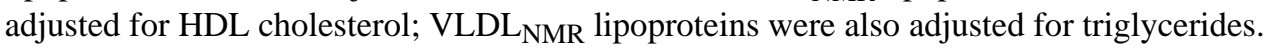
Each multivariable logistic model examining the association between a NMR lipoprotein and recurrent PTB had good model fit as indicted by the Hosmer-Lemeshow test. None of the interactions between NMR lipoproteins and treatment group was significant.

\section{LDL $L_{\text {NMR }}$ lipoproteins}

None of the $\mathrm{LDL}_{\mathrm{NMR}}$ lipoprotein concentrations or particle size was associated with recurrent PTB (Table 2).

\section{HDL $L_{\text {NMR }}$ lipoproteins}

After adjusting for confounders and standard HDL cholesterol, non-linear trends were observed for two $\mathrm{HDL}_{\mathrm{NMR}}$ lipoproteins, total $\mathrm{HDL}_{\mathrm{NMR}}$ particle concentration and medium HDL $_{\text {NMR }}$ particle concentration (Table 2). An inverted U-shaped trend was observed for total $\mathrm{HDL}_{\mathrm{NMR}}$ particle concentration (linear term: $\beta=0.528 ; \mathrm{SE}=0.235 ; \mathrm{p}=0.02$, quadratic term: $\beta=-0.007 ; \mathrm{SE}=0.003 ; \mathrm{p}=0.03$ for the odds of recurrent PTB). For medium HDL $\mathrm{NMR}_{\mathrm{N}}$ particle concentration, an irregularly shaped tend was observed with an increased odds of $2.88(\mathrm{CI}=1.17-7.11 ; \mathrm{p}=0.02)$ for recurrent PTB in tertile 2 compared with tertile 1 . These trends did not reach statistical significance $<0.01$.

\section{VLDL $_{\text {NMR }}$ lipoproteins}

After adjusting for confounders and standard triglycerides, an association was observed for VLDL $_{\text {NMR }}$ particle size (Table 2). A linear trend was observed, with an increased odds of recurrent PTB of 1.04 (95\% CI=1.01-1.08; $\mathrm{p}=0.02)$ per nanometer increase in $\mathrm{VLDL}_{\mathrm{NMR}}$ particle size. When examining tertiles, increased odds of recurrent PTB was observed for the $3^{\text {rd }}$ tertile of $\mathrm{VLDL}_{\mathrm{NMR}}$ particle size compared with the $1^{\text {st }}$ tertile $(\mathrm{OR}=3.00 ; 95 \% \mathrm{CI}=1.40$ 6.43; $\mathrm{p}=0.005$ ) (Table 2).

\section{DISCUSSION}

In this exploratory analysis, variations in mid-pregnancy lipoproteins were not associated with recurrent PTB overall, however the trend observed with increasing VLDL NMR $_{\text {particle }}$ size and increasing odds of recurrent PTB was suggestive. Most importantly, LDL concentration appears to have no role in recurrent PTB, unlike the crucial role this lipoprotein plays in adult cardiovascular disease.

Pregnancy is a profound vascular event with uterine blood flow being increased from $<1 \%$ to $10-15 \%$ of cardiac output via remodeling of the utero-placental vasculature.

Atherosclerotic-like lesions with cholesterol-laden macrophages in the maternal spiral arteries have been found in pathologic specimens from pregnancies complicated by PTB, fetal growth restriction, and preeclampsia (9). If the vascular biology at the utero-placental interface duplicates that seen in other vascular beds, then LDL and VLDL particles play a major role in cholesterol transport into the lesions and HDL particles help cholesterol efflux. Extrapolating further, particle size and number of lipoproteins as detected by NMR may influence the development and regression of these lesions $(15,16)$.

VLDL carries the bulk of triglycerides (TG) within the circulations of pregnancy and nonpregnant women. Larger particles are more TG rich and the concentration of VLDL rises progressively as pregnancy advances (6). This phenomenon is thought to be due to the 
insulin resistance induced by pregnancy and is critical to create an energy-rich environment within the maternal circulation to serve the caloric needs of the developing fetus (1). Our observed trend of increased frequency of recurrent PTB with increasing VLDL particle size (the larger the particle the greater the magnitude of risk) has heretofore not been described.

HDL plays a crucial role in the transport of lipids out of vessel walls in non-pregnant adults (15). This lipoprotein increases along with other lipoproteins in pregnancy. The particle is decreased as insulin resistance worsens (1). The smaller the HDL particle the less effective that particle is in protecting against cardiovascular disease (15). Conversely, to the case in cardiovascular epidemiology where HDL levels are inversely proportional to risk of disease, we observed an inverted U-shaped relationship, albeit non-significant per $\mathrm{P}<0.01$ criterion, between total HDL particle size and recurrent PTB.

Our findings from this secondary exploratory analysis of a clinical trial can at most suggest hypothetical pathways for future investigations. All of our case and control patients received progesterone therapy which could have altered lipid metabolism or influenced the results by other mechanisms. It does demonstrate that lipid biology in pregnancy is complex and advances in cardiovascular biology cannot be easily extrapolated to the pathogenesis of poor pregnancy outcome. Ideally, our study would have been done with fasting samples to avoid any effects of feeding on triglycerides and the particles that transport triglycerides. Some recent papers question the utility of fasting in lipid assessments and have concluded that fasting is not helpful $(17,18,19)$. Additionally, given the number of lipid biomarkers assessed (seventeen), the association between VLDL particle size and recurrent PTB our findings may simply reflect a type-1 statistical error. Also we only measured lipoproteins at mid-pregnancy and earlier or later assessments could provide different results. Given the many lifestyle, nutritional, and pharmaceutical options available to alter lipid profiles, this remains an area worthy of more study.

\section{Acknowledgments}

The project described was supported by grants from the Eunice Kennedy Shriver National Institute of Child Health and Human Development (NICHD) [HD27860, HD27917, HD40560, HD34208, HD40485, HD21410, HD27915, HD40500, HD40512, HD40544, MO1-RR-000080, HD34136, HD27869, HD40545, HD36801, HD19897] and does not necessarily represent the official views of the NICHD or NIH.

\section{Appendix}

In addition to the authors, other members of the Eunice Kennedy Shriver National Institute of Child Health and Human Development Maternal-Fetal Medicine Units Network are as follows:

University of North Carolina at Chapel Hill, Chapel Hill, NC- K. Dorman, E. Prata, K. Hamden

Wake Forest University Health Sciences, Winston-Salem, NC- P. Meis, M. Swain, B. Scott, C. Leftwich

Wayne State University, Detroit, MI- G. Norman, D. Driscoll, C. Sudz, L. Wynn, S. Blackwell

University of Utah Health Sciences Center, Salt Lake City, UT - K. Anderson (University of Utah Health Sciences Center), S. Bonnemort (McKay-Dee Hospital), D. Lund (University of Utah Health Sciences Center), J. Russell (LDS Hospital), J. Parsons (Utah Valley Regional Medical Center) 
Columbia University, New York, $N Y$ - S. Bousleiman, S. South, V. Carmona, H. Husami, C. Lankford, C. Perez

University of Pittsburgh, Pittsburgh, PA- M. Luce, M. Cotroneo

The Ohio State University, Columbus, $\mathrm{OH}-$ F. Johnson, M. Landon, D. Cline, H. Walker

Women and Infants Hospital, Brown University, Providence, RI- M. Carpenter, D. Allard, J. Tillinghast

Northwestern University, Chicago, IL - M. Dinsmoor (Evanston Hospital), P.J. Simon, M. Huntley, C. Whitaker-Carr, M. Ramos-Brinson, G. Mallett

Case Western Reserve University-MetroHealth Medical Center, Cleveland, OH-C. Milluzzi, J. Hunter, W. Dalton, H. Ehrenberg, B. Stetzer

Drexel University College of Medicine- M. Hoffman, M. Talucci, C. Tocci, S. Wilson, M. Lake

University of Alabama at Birmingham, Birmingham, $A L-\mathrm{W}$.W. Andrews, A. Northen, M. Parks, P. Blake Files

The University of Texas Health Science Center at Houston, Houston, TX - L.C. Gilstrap, B. Glenn-Cole, K. Cannon

The George Washington University Biostatistics Center- E. Thom, P. McGee, J. Zachary, R. Palugod, L. Leuchtenburg

Eunice Kennedy Shriver National Institute of Child Health and Human Development, Bethesda, $M D-$ C. Spong, S. Tolivaisa

\section{REFERENCES}

1. Huda SS, Sattar N, Freeman DJ. Lipoprotein metabolism and vascular complications in pregnancy. Clinical Lipidology. 2009; 4:91-102.

2. Ramsay JE, Ferrell WR, Crawford L, Wallace AM, Greer IA, Sattar N. Maternal obesity is associated with dysregulation of metabolic, vascular, and inflammatory pathways. J Clin Endocrinol Metab. 2002; 87:4231-4237. [PubMed: 12213876]

3. O'Brien TE, Ray JG, Chan WS. Maternal body mass index and the risk of preeclampsia: a systematic overview. Epidemiology. 2003; 14:368-374. [PubMed: 12859040]

4. Torloni MR, Betrán AP, Horta BL, et al. Prepregnancy BMI and the risk of gestational diabetes: a systematic review of the literature with meta-analysis. Obes Rev. 2009; 10:194-203. [PubMed: 19055539]

5. Rizzo M, Berneis K, Altinova AE, et al. Atherogenic lipoprotein phenotype and LDL size and subclasses in women with gestational diabetes. Diabet Med. 2008; 25:1406-1411. [PubMed: 19046238]

6. Mackay VA, Huda SS, Stewart FM, et al. Preeclampsia is associated with compromised maternal synthesis of long-chain polyunsaturated fatty acids, leading to offspring deficiency. Hypertension. 2012; 60:1078-1085. [PubMed: 22949531]

7. Nielsen LB. Transfer of low density lipoprotein into the arterial wall and risk of atherosclerosis. Atherosclerosis. 1996; 123:1-15. [PubMed: 8782833]

8. Tabas I, Williams KJ, Borén J. Subendothelial lipoprotein retention as the initiating process in artherosclerosis: update and therapeutic implications. Circulation. 2007; 116:1832-1844. [PubMed: 17938300] 
9. Basaran A. Pregnancy-induced hyperlipoproteinemia: review of the literature. Reprod Sci. 2009; 16:431-437. [PubMed: 19233944]

10. Sattar N, Greer IA, Louden J, et al. Lipoprotein subfraction changes in normal pregnancy: threshold effect of plasma triglyceride on appearance of small, dense low density lipoprotein. $\mathrm{J}$ Clin Endocrinol Metab. 1997; 82:2483-2491. [PubMed: 9253322]

11. Lau JF, Smith DA. Advanced lipoprotein testing: recommendations based on current evidence. Endocrinol Metab Clin North Am. 2009; 38:1-31. [PubMed: 19217510]

12. Harper M, Thom E, Klebanoff MA, et al. Omega-3 fatty acid supplementation to prevent recurrent preterm birth; a randomized controlled trial. Obstet Gynecol. 2010; 115:234-242. [PubMed: 20093894]

13. Brunzell JD, Davidson M, Furberg CD, et al. American Diabetes Association; American College of Cardiology Foundation. Lipoprotein management in patients with cardiometabolic risk: consensus statement from the American Diabetes Association and the American College of Cardiology Foundation. Diabetes Care. 2008; 31:811-822. [PubMed: 18375431]

14. Jeyarajah EJ, Cromwell WC, Otvos JD. Lipoprotein particle analysis by nuclear magnetic resonance spectroscopy. Clin Lab Med. 2006; 26:847-870. [PubMed: 17110242]

15. Meurs I, Van Eck M, Van Berkel TJ. High-density lipoprotein: key molecule in cholesterol efflux and the prevention of atherosclerosis. Curr Pharm Des. 2010; 16:1445-1467. [PubMed: 20199377]

16. Mumford SL, Schisterman EF, Siega-Riz AM, et al. A longitudinal study of serum lipoproteins in relation to endogenous reproductive hormones during the menstrual cycle: findings from the BioCycle study. J Clin Endocrinal Metab. 2010; 95:E80-E85.

17. Sidhu D, Naugler C. Fasting time and lipid levels in a community-based population: a crosssectional study. Arch Intern Med. 2012; 172:1707-1710. [PubMed: 23147400]

18. Gaziano JM. Should we fast before we measure our lipids? Arch Internal Med. 2012; 172:17041706.

19. Khera AV, Mora S. Fasting for lipid testing: Is it worth the trouble? Arch Intern Med. 2012; 172:1710-1712. 
Table 1

Characteristics of the study population

\begin{tabular}{|c|c|c|c|}
\hline & $\begin{array}{c}\text { Cases } \\
\begin{array}{c}\text { (recurrent PTB } \\
\text { weeks) }\end{array}\end{array}$ & $\begin{array}{c}\text { Controls } \\
\text { (term birth } \geq 37 \\
\text { weeks) }\end{array}$ & P-value \\
\hline $\mathrm{N}$ & 128 & 132 & \\
\hline Age (years), mean $\pm \mathrm{SD}$ & $26.9 \pm 5.5$ & $27.1 \pm 5.5$ & 0.80 \\
\hline Race/ethnicity, N (\%) & & & 0.82 \\
\hline Non- Hispanic Black & $52(40.6)$ & $50(37.9)$ & \\
\hline Hispanic & $15(11.7)$ & $14(10.6)$ & \\
\hline Non- Hispanic White & $61(47.7)$ & $68(51.5)$ & \\
\hline Pre-pregnancy body mass index $\left(\mathrm{kg} / \mathrm{m}^{2}\right)$, mean $\pm \mathrm{SD}$ & $26.9 \pm 7.2$ & $26.4 \pm 6.2$ & 0.82 \\
\hline Smokers, N (\%) & $32(25.0)$ & $13(9.9)$ & 0.002 \\
\hline Preeclampsia or gestational hypertension, $\mathrm{N}(\%)$ & $5(3.9)$ & $4(3.1)$ & 0.75 \\
\hline Assigned to omega-3 group, $\mathrm{N}(\%)$ & $61(47.7)$ & $71(53.8)$ & 0.39 \\
\hline Number of prior preterm deliveries, $\mathrm{N}(\%)$ & & & 0.001 \\
\hline 1 & $78(60.9)$ & $106(80.3)$ & \\
\hline 2 or more & $50(39.1)$ & $26(19.7)$ & \\
\hline Total cholesterol, $\mathrm{mg} / \mathrm{dl}$, median (interquartile range) & $203(181,229)$ & $212(186,238)$ & 0.12 \\
\hline \multicolumn{4}{|l|}{ LDL measures } \\
\hline Standard LDL cholesterol, direct, $\mathrm{mg} / \mathrm{dl}$, median (interquartile range) & $97(82,110)$ & $103(81,120)$ & 0.21 \\
\hline \multicolumn{4}{|l|}{$\begin{array}{l}\mathrm{LDL}_{\mathrm{NMR}} \text { lipoprotein particle concentration, nmol/l, median (interquartile } \\
\text { range) }\end{array}$} \\
\hline Total & $1114.5(897.0,1401.0)$ & $1154.0(856.5,1365.5)$ & 0.89 \\
\hline Large & $636.5(497.5,811.5)$ & $684.0(506.0,841.0)$ & 0.26 \\
\hline Medium small & $70.0(6.5,148.0)$ & $47.0(9.0,124.0)$ & 0.57 \\
\hline Small & $376.5(26.0,767.5)$ & $285.0(57.0,624.5)$ & 0.55 \\
\hline Very small & $309.5(23.5,613.5)$ & $212.0(29.5,513.5)$ & 0.53 \\
\hline IDL & $46.5(15.0,81.0)$ & $46.5(9.0,90.5)$ & 0.94 \\
\hline $\mathrm{LDL}_{\mathrm{NMR}}$ average particle size, $\mathrm{nm}$, median (interquartile range) & $21.9(21.2,22.6)$ & $22.1(21.5,22.6)$ & 0.34 \\
\hline \multicolumn{4}{|l|}{ HDL measures } \\
\hline Standard HDL, mg/dl, median (interquartile range) & $58(51,64)$ & $60(52,68)$ & 0.11 \\
\hline \multicolumn{4}{|l|}{$\begin{array}{l}\mathrm{HDL}_{\mathrm{NMR}} \text { lipoprotein particle concentration, } \mu \mathrm{mol} / \mathrm{l} \text {, median (interquartile } \\
\text { range) }\end{array}$} \\
\hline Total & $32.9(29.9,36.8)$ & $32.9(29.9,36.6)$ & 0.88 \\
\hline Large & $11.8(9.5,13.9)$ & $11.6(10.0,13.3)$ & 0.93 \\
\hline Medium & $0.1(0.0,2.1)$ & $0.0(0.0,1.6)$ & 0.16 \\
\hline Small & $19.4(17.4,22.3)$ & $20.2(17.0,23.0)$ & 0.73 \\
\hline $\mathrm{HDL}_{\mathrm{NMR}}$ average particle size, $\mathrm{nm}$, median (interquartile range) & $9.8(9.5,10.0)$ & $9.8(9.6,10.0)$ & 0.23 \\
\hline \multicolumn{4}{|l|}{ VLDL measures } \\
\hline Standard triglycerides, $\mathrm{mg} / \mathrm{dl}$ & $157(125,214)$ & $148(111,196)$ & 0.17 \\
\hline
\end{tabular}




\begin{tabular}{|lcccc|}
\hline & $\begin{array}{c}\text { Cases } \\
\text { (recurrent PTB }<35 \\
\text { weeks) }\end{array}$ & $\begin{array}{c}\text { Controls } \\
\text { (term birth } \geq \mathbf{3 7} \\
\text { weeks) }\end{array}$ & P-value $^{\boldsymbol{a}}$ \\
\hline VLDL $_{\mathrm{NMR}}$ lipoprotein particle concentration, $\mathrm{nmol} / \mathrm{l}$ & $70.1(53.2,93.4)$ & $74.4(47.1,101.4)$ & 0.79 \\
\hline Total VLDL/chylomicrons & $2.3(0.6,4.8)$ & $1.4(0.5,3.4)$ & 0.04 \\
\hline Large VLDL/chylomicrons & $26.6(16.2,38.4)$ & $27.8(15.1,39.8)$ & 0.83 \\
\hline Medium & $41.2(28.4,54.2)$ & $42.4(26.8,61.4)$ & 0.39 \\
\hline Small & $50.7(45.9,56.9)$ & $47.6(43.7,53.1)$ & 0.003 \\
\hline VLDL $_{\mathrm{NMR}}$ average particle size, $\mathrm{nm}$ & & & & \\
\hline
\end{tabular}

PTB, preterm birth; LDL, low density lipoproteins; NMR, nuclear magnetic resonance; IDL, intermediate density lipoproteins; HDL, high density lipoproteins; VLDL, very low density lipoproteins.

${ }^{a}$ Continuous variables were compared using the Wilcoxon test; categorical variables were compared using the chi-square or Fisher's exact test 


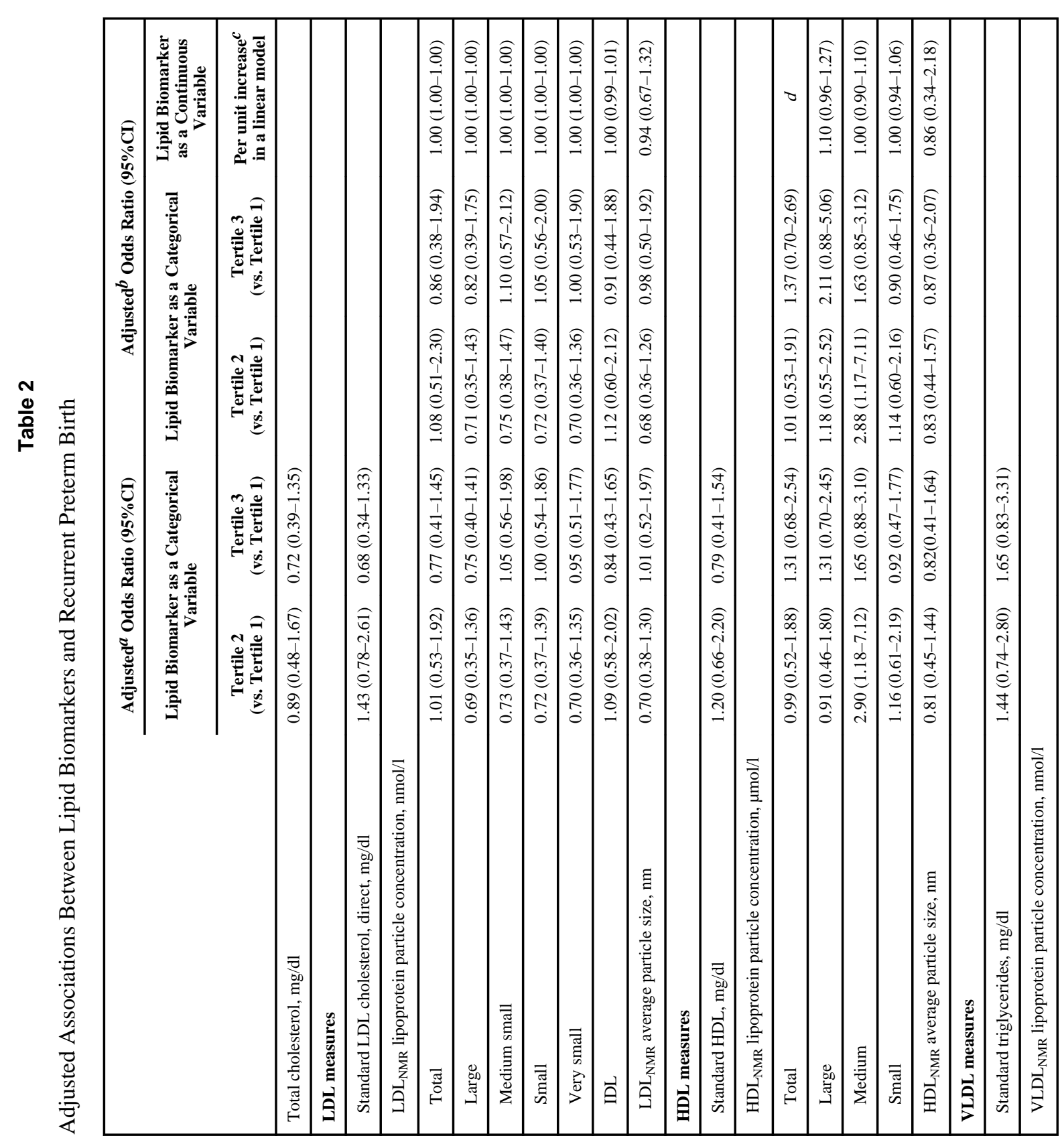

Am J Obstet Gynecol. Author manuscript; available in PMC 2014 October 01. 


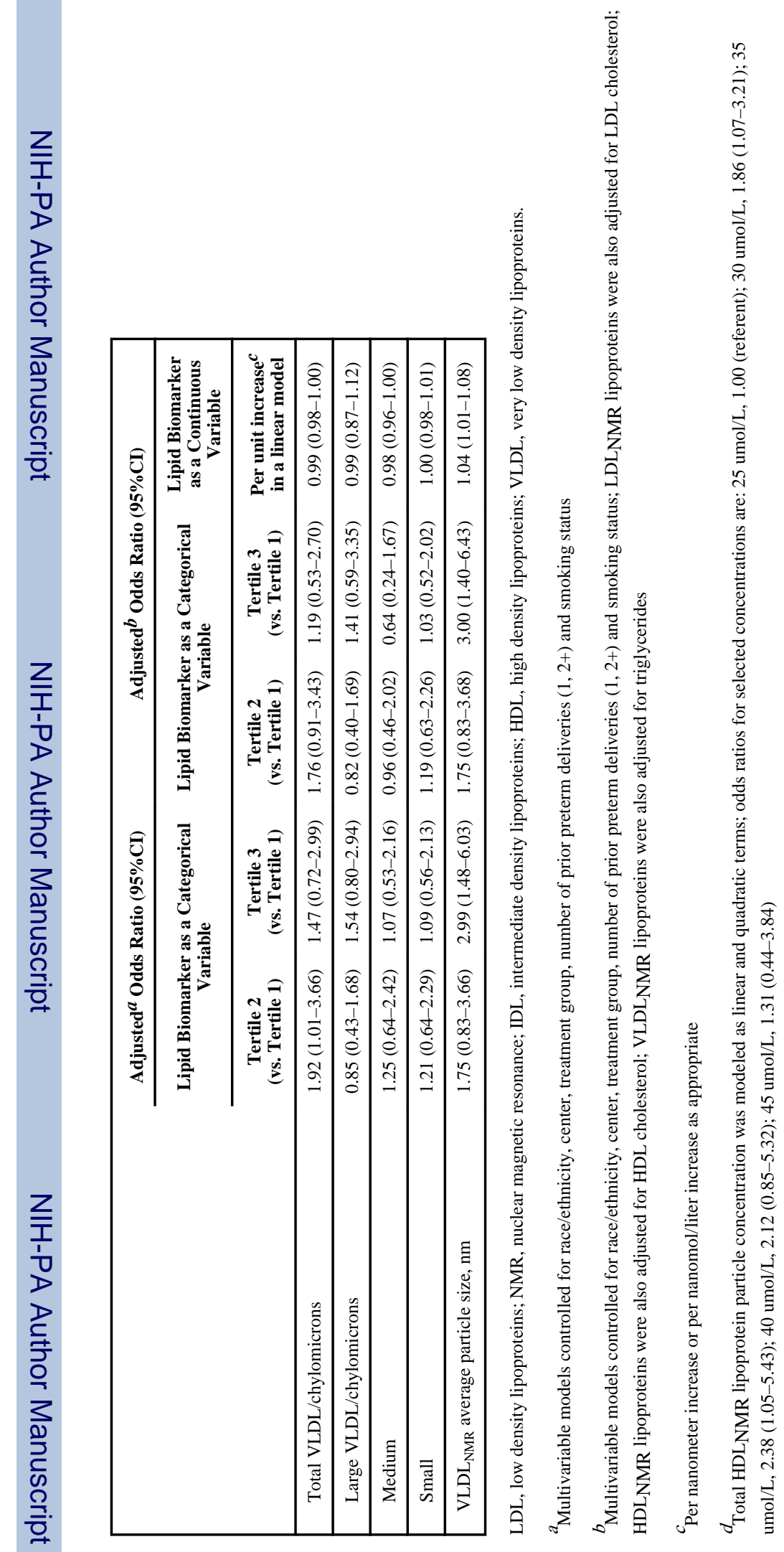

